\title{
Molecular targets of natural health products in arthritis
}

\author{
Sarah Khalifé and Muhammad Zafarullah* \\ See related research by Rasheed et al., http://arthritis-research.com/content/12/5/R195
}

Abstract
Patients with rheumatoid arthritis (RA) and
osteoarthritis (OA) consume'natural health products'
(NHPs) whose therapeutic efficacy, toxicity and
mechanisms of action are poorly understood. In
a previous issue of Arthritis Research and Therapy,
Haqqi and colleagues characterized IL-1-activated
mitogen-activated protein kinase kinase 3 (MKK3)
and p38-mitogen-activated protein kinase (MAPK)
isoforms in human OA chondrocytes. The cartilage-
protective mechanisms of pomegranate extract involve
diminishing MKK3-activated p38a, JNK, NF-KB and
Runx2 pathways, which regulate inflammatory proteins
and cartilage-destroying proteases. Epigallocatechin-
3-gallate, resveratrol, curcumin and other NHP active
ingredients suppress multiple inflammatory and
catabolic molecular mediators of arthritis. Non-toxicity,
reduced severity and incidence of arthritis in animal
models warrant testing NHP active ingredients for
preventing human OA and RA.

Rheumatoid arthritis (RA) and osteoarthritis (OA) cause enormous patient suffering and economic burden. Due to non-steroidal anti-inflammatory drug (NSAID)- and cyclooxygenase-2 (COX-2) inhibitor-associated ulcers and cardiovascular adverse events, novel anti-arthritis therapies are needed. Natural health product (NHP) use by patients to alleviate symptoms is rising globally. However, the quality of NHPs is poorly regulated and their efficacy, toxicity and mechanism(s) of action are not understood. Using state-of-the-art approaches, Haqqi and colleagues report in Arthritis Research and Therapy [1] that human chondrocytes expressed the p38-mitogenactivated protein kinase (MAPK) isoforms $\mathrm{p} 38 \alpha,-\gamma$ and

*Correspondence: Muhammad.Zafarullah@umontreal.ca

Department of Medicine, University of Montreal and CRCHUM, Hôpital NotreDame, K-5255 Mailloux, 1560 Sherbrooke est, Montréal, Québec, Canada H2L 4M1 $-\delta$, but not $\mathrm{p} 38 \beta$. IL-1 $\beta$ enhanced phosphorylation of p38 $\alpha$-MAPK and p38 $\gamma$-MAPK but not of p38 $\delta$-MAPK. They showed by gene-silencing that p38-MAPK activation was mediated by mitogen-activated protein kinase kinase 3 (MKK3). A standardized pomegranate extract (PE) specifically diminished IL-1-induced and MKK3activated $\mathrm{p} 38 \alpha$-MAPK isoform and Runx2 (runt-related transcription factor 2) activity. PE did not interfere with the binding of IL- $1 \beta$ to IL-1 receptor, thus ruling out steric hindrance as a suppression mechanism. PE also inhibited JNK-MAPK and NF- $\mathrm{KB}$ activity.

p38, JNK-MAPK and NF-kB pathways are also major targets of drug discovery in arthritis. The p38 cascade regulates proinflammatory genes post-transcriptionally but remains a controversial target due to modest efficacy and severe toxicity in clinical trials [2]. The NF-kB pathway regulates cytokine and ADAMTS- 4 genes, and is an important therapeutic target in arthritis [3]. Activated JNK and c-Jun constitute AP-1 factor, which enhances synovitis in RA and matrix metalloproteinase (MMP)mediated cartilage loss [4]. Runx2 regulates induction of collagen II-destroying human MMP-13 and aggrecandegrading, ADAMTS (a disintegrin and metalloproteinase with thrombospondin motifs) genes [5], whose inhibition by PE could potentially reduce cartilage degradation. Runx2-deficient (heterozygous) mice with surgically induced knee OA displayed reduced cartilage destruction, osteophyte formation, type X collagen and MMP-13 expression (features of OA-associated aberrant hypertrophic chondrocyte differentiation) versus wild-type mice [6]. Thus, beneficial effects of PE may be through these pharmacological targets.

Since the gastrointestinal tract may degrade the active ingredients of NHPs, Haqqi and colleagues [7] demonstrated that oral administration of PE in collagen-induced arthritis mouse model protects joints from inflammatory arthritis. The chondroprotective effects of another PE were reconfirmed in the monoiodoactate-induced OA model [8]. These studies reveal molecular mechanisms of PE action, including cartilage-protective active compounds (polyphenols, ellagitannins, quercetin, gallic acid and ellagic acid) that work through precise targets [7]. 
Considering the non-toxicity of $\mathrm{PE}$ in chondrocytes and its effectiveness in RA and OA models, identifying precise pomegranate active ingredients, their synergistic effects and utility for preventing or reducing human OA and RA through clinical trials is warranted. Epidemiological studies on non-consuming and pomegranateconsuming populations for incidence of arthritis may also be useful. One should also analyze potential pharmacological drug-PE interactions.

The anti-arthritic effects of green tea are attributed to the multiple activities of non-toxic epigallocatechin-3gallate (EGCG). It suppresses IL-1-induced glycosaminoglycan release from cartilage by inhibiting ADAMTS, MMP-1 and MMP-13 expression through preferentially blocking NF- $\mathrm{kB}$ activity in chondrocytes. EGCG also inhibits IL-1-stimulated inducible nitric oxide synthase (iNOS), nitric oxide and JNK activities, which mediate cartilage degradation. In RA synovial fibroblasts, EGCG inhibits TNF- $\alpha$-induced MMP-1, MMP-3, ERK (extracellular signal-regulated kinase), p38, JNK and AP-1 activities. EGCG sensitizes RA fibroblasts to TNF- $\alpha$ induced caspase-3-mediated apoptosis by blocking Akt and NF- $\kappa B$ cell survival pathways. EGCG also inhibits IL-6 receptor-induced MMP-2, increases gp130 receptor in fibroblasts and ameliorates adjuvant-induced rat arthritis. EGCG suppresses osteoclast-specific, NF-ATc1 and RA-associated bone resorption and ameliorates mouse arthritis. It inhibited oncostatin M-stimulated CCL2 chemokine expression in human osteoblasts and reduced severity of collagen-induced arthritis. These in vitro and in vivo studies suggest that EGCG could reduce synovial hyperplasia, cartilage degradation and bone resorption by modulating multiple targets in joints [9]. Despite its apparent safety in clinical trials of other diseases, its potential for causing apoptosis in chondrocytes should be tested further.

Resveratrol from grapes displays anti-inflammatory and chondroprotective activities by increasing proteoglycan synthesis and chondrocyte proliferation and by suppressing IL-1, reactive oxygen species, p53-induced apoptosis, leukotriene B4, prostaglandin E2 synthesis and MMPs in vitro [10]. Intra-articular resveratrol treatment reduced cartilage loss in rabbit arthritis models. Resveratrol induced fibroblast apoptosis but increased chondrocyte survival by activating Sirtuin 1 (a histone deacetylase) and Bcl2 [11,12]. Thus, resveratrol may prevent intervertebral disc degeneration, $\mathrm{OA}$-associated inflammation, chondrocyte apoptosis, and RA-related pannus formation, desirable goals in treating OA and RA. Resveratrol-like Sirtuin 1 activators are also being developed as lifespan-extending drugs.

Curcumin suppresses inflammatory and catabolic mediators such as IL-1 $\beta$-stimulated nitric oxide, prostaglandin E2, COX-2, IL-6, IL-8, MMP-3, MMP-9, JNK and
NF- $\mathrm{kB}$ activation and the oncostatin M-induced JAK/ STAT pathway in human chondrocytes [13]. It promotes chondrogenesis from mesenchymal stem cells by antagonizing proinflammatory cytokines [14]. Despite these anti-inflammatory, antioxidant, anti-angiogenic activities, its oral consumption and tissue bioavailability are problematic. Other NHPs, quercetin, triptolide and silymarin, target inflammatory cytokines, NF- $\mathrm{kB}, \mathrm{COX}-2$ and catabolic MMPs [15].

It is laudable that regulatory agencies are encouraging research to substantiate the therapeutic claims by manufacturers of NHPs. We need well-controlled, molecular and mechanistic studies on NHPs as modeled by Haqqi and colleagues [1]. Currently, we can analyze multiple targets of NHPs by gene arrays and proteomics to evaluate their efficacy in preventing and treating RA and OA.

\section{Abbreviations}

ADAMTS, a disintegrin and metalloproteinase with thrombospondin motifs; COX-2, cyclooxygenase-2; EGCG, epigallocatechin-3-gallate; IL, interleukin; JNK, c-Jun N-terminal kinase; MAPK, mitogen-activated protein kinase; MKK3, mitogen-activated protein kinase kinase 3; MMP, matrix metalloproteinase; $\mathrm{NF}$, nuclear factor; $\mathrm{NHP}$, natural health product; $\mathrm{OA}$, osteoarthritis; $\mathrm{PE}$, pomegranate extract; RA, rheumatoid arthritis; TNF, tumor necrosis factor.

\section{Competing interests}

The authors declare that they have no competing interests.

\section{Acknowledgements}

The work in our laboratory was supported by the Canadian Institutes of Health Research.

Published: 3 February 2011

\section{References}

1. Rasheed Z, Akhtar N, Haqqi TM: Pomegranate extract inhibits the interleukin-1 beta-induced activation of MKK-3, p38alpha-MAPK and transcription factor RUNX-2 in human osteoarthritis chondrocytes. Arthritis Res Ther 2010, 12:R195.

2. Genovese MC: Inhibition of $\mathrm{p} 38$ : has the fat lady sung? Arthritis Rheum 2009, 60:317-320.

3. Roman-Blas JA, Jimenez SA: Targeting NF-kappaB: a promising molecular therapy in inflammatory arthritis. Int Rev Immunol 2008, 27:351-374.

4. Svensson Cl, Inoue T, Hammaker D, Fukushima A, Papa S, Franzoso G, Schett G, Corr M, Boyle DL, Firestein GS: Gadd45beta deficiency in rheumatoid arthritis: enhanced synovitis through JNK signaling. Arthritis Rheum 2009, 60:3229-3240.

5. Thirunavukkarasu K, Pei Y, Moore TL, Wang H, Yu XP, Geiser AG, Chandrasekhar $S$ : Regulation of the human ADAMTS-4 promoter by transcription factors and cytokines. Biochem Biophys Res Commun 2006, 345:197-204.

6. Kamekura S, Kawasaki Y, Hoshi K, Shimoaka T, Chikuda H, Maruyama Z, Komori T, Sato S, Takeda S, Karsenty G, Nakamura K, Chung UI, Kawaguchi H: Contribution of runt-related transcription factor 2 to the pathogenesis of osteoarthritis in mice after induction of knee joint instability. Arthritis Rheum 2006, 54:2462-2470.

7. Shukla M, Gupta K, Rasheed Z, Khan KA, Haqqi TM: Consumption of hydrolyzable tannins-rich pomegranate extract suppresses inflammation and joint damage in rheumatoid arthritis. Nutrition 2008, 24:733-743.

8. Hadipour-Jahromy M, Mozaffari-Kermani R: Chondroprotective effects of pomegranate juice on monoiodoacetate-induced osteoarthritis of the knee joint of mice. Phytother Res 2010, 24:182-185.

9. Ahmed S: Green tea polyphenol epigallocatechin 3-gallate in arthritis: progress and promise. Arthritis Res Ther 2010, 12:208.

10. Csaki C, Keshishzadeh N, Fischer K, Shakibaei M: Regulation of inflammation signalling by resveratrol in human chondrocytes in vitro. Biochem 
Pharmacol 2008, 75:677-687.

11. Byun HS, Song JK, Kim YR, Piao L, Won M, Park KA, Choi BL, Lee H, Hong JH, Park J, Seok JH, Lee YJ, Kang SW, Hur GM: Caspase-8 has an essential role in resveratrol-induced apoptosis of rheumatoid fibroblast-like synoviocytes. Rheumatology (Oxford) 2008, 47:301-308.

12. Takayama K, Ishida K, Matsushita T, Fujita N, Hayashi S, Sasaki K, Tei K, Kubo S, Matsumoto T, Fujioka H, Kurosaka M, Kuroda R: SIRT1 regulation of apoptosis of human chondrocytes. Arthritis Rheum 2009, 60:2731-2740.

13. Henrotin Y, Clutterbuck AL, Allaway D, Lodwig EM, Harris P, Mathy-Hartert M, Shakibaei M, Mobasheri A: Biological actions of curcumin on articular chondrocytes. Osteoarthritis Cartilage 2010, 18:141-149.

14. Buhrmann C, Mobasheri A, Matis U, Shakibaei M: Curcumin mediated suppression of nuclear factor-kappaB promotes chondrogenic differentiation of mesenchymal stem cells in a high-density co-culture microenvironment. Arthritis Res Ther 2010, 12:R127.

15. Khanna D, Sethi G, Ahn KS, Pandey MK, Kunnumakkara AB, Sung B, Aggarwal A, Aggarwal BB: Natural products as a gold mine for arthritis treatment. Curr Opin Pharmacol 2007, 7:344-351.

doi:10.1186/ar3222

Cite this article as: Khalifé S, Zafarullah M: Molecular targets of natural

health products in arthritis. Arthritis Research \& Therapy 2011, 13:102 